\title{
Instant Theft Alert and Tracking System in Car
}

\author{
Kunjal P. Tanna \\ Final Year Student, Electronics \\ and Telecommunication \\ 23/24, Manusmruti, R.R.T. Road, \\ Mulund (W), Mumbai-400080
}

\author{
Preeti Kumar \\ Final Year Student, Electronics \\ and Telecommunication \\ 10-C Kanchanjunga, \\ Anushaktinagar, Mumbai-400094
}

\author{
Shubha Narayanan \\ Final Year Student, Electronics \\ and Telecommunication \\ 303, Amol Deep, Mhatre Nagar, \\ Rajaji Path, Dombivli(E) 421201
}

\begin{abstract}
There are a variety of areas wherein a need exists for a system capable of identifying and tracking the geographic location of a remote valuable object. One such valuable object which requires constant surveillance is car. In order to alert the car owner immediately regarding theft of the car by means of a portable remote receiver, we have come up with an Event Tracking System, which we have discussed in this paper. This system consists of Event Detection Module, Global Positioning System (GPS), Asset Tag and Wireless Network Interface. The Event Detection Module consists of Event detection sensor and Event detection logic. GPS and Asset Tag together determine the location of the car. Wireless Network Interface consists of a transmitter used to transmit signal to the Surveillance Pad wirelessly. The Surveillance Pad consists of processing unit, display and alert system. The predetermined event to be detected in this system is the unlocking of the door of the car. Once the door gets unlocked it will send an alert to the car owner in the form of an alarm or message. This can help the user to take immediate actions. Once the engine starts, the GPS tracking display in the Surveillance Pad will get enabled with an alarm allowing the car owner to track the car and retrieve it.
\end{abstract}

\section{INTRODUCTION}

Presently, object tracking is widely used for tracking the stolen objects. But this method fails to immediately inform the user regarding the theft. Thus, delayed knowledge regarding theft makes the process of retrieving the object difficult. Hence, it would be more advantageous if the theft is detected instantly. So we have come up with a system which initially detects the event further tracking it.

According to the Federal Bureau of Investigation (FBI)'s Uniform Crime Reports, a motor vehicle is stolen in the United States every 26.4 seconds. The odds of a vehicle being stolen were 1 in 207 in 2005 (data available based on registrations from the Federal Highway Administration, thefts from the FBI and calculated by the Insurance Information Institute). The odds are highest in urban areas. Total numbers of more than 40,000 cars, costing about 160 crore, are stolen every year in India. On an average, over the past five years, 2,500 vehicles were stolen annually, but the figures have zoomed to 3,282 in the past twelve months. The above statistics show that there is an urgent need to implement a system that immediately alerts the car owner about the theft followed by its tracking.

\section{GENERAL PROCEDURE}

The general procedure followed by our system is described in Figure 1:

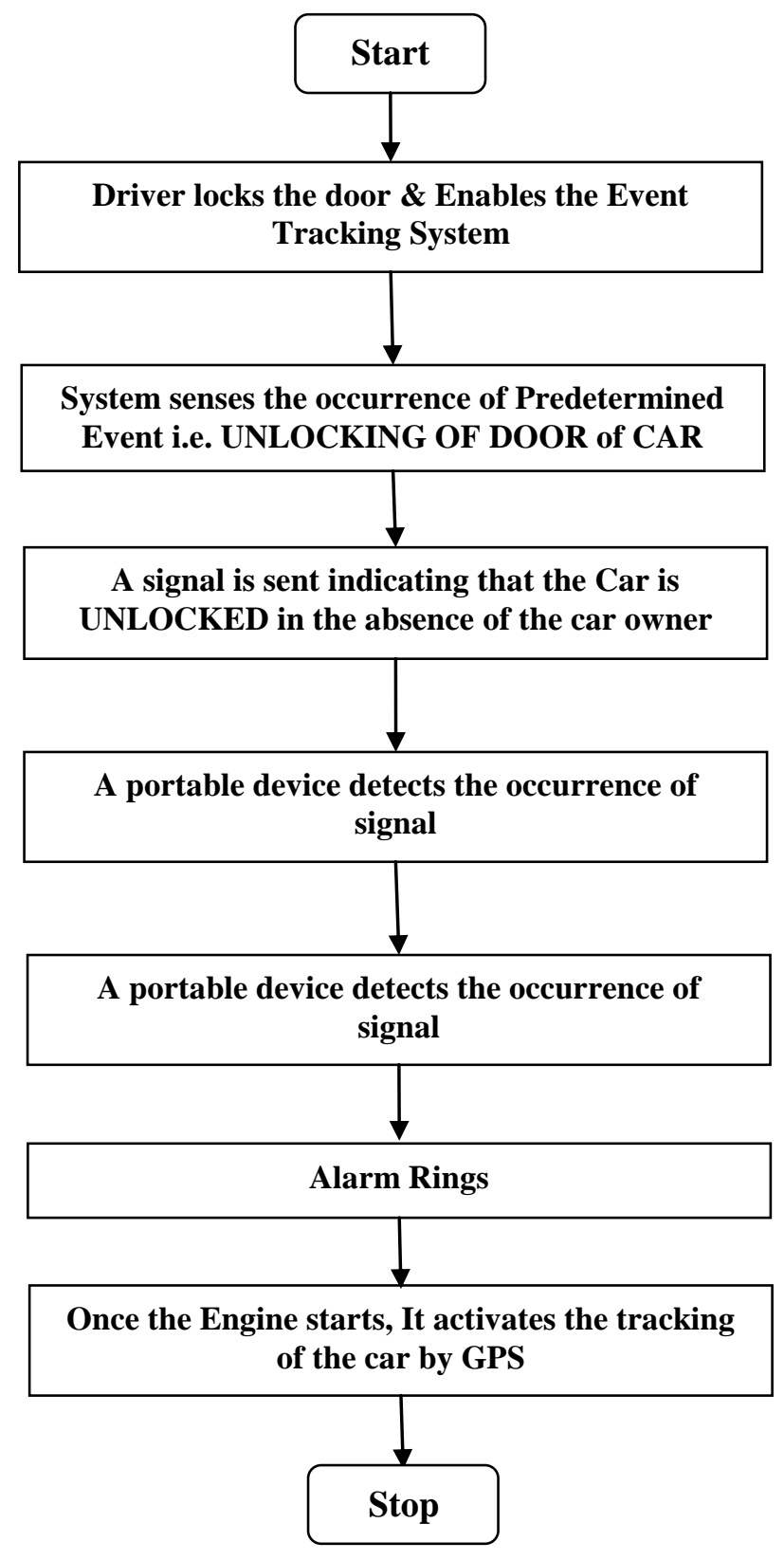

Figure 1: Flowchart of General Procedure 
Driver locks the door and enables the system. This avoids unwanted alerts while the owner uses the car. The car owner carries a portable device which has an alarm system and a tracking system which communicates with the security system installed in the car. As soon as the thief tries to unlock the door, the security system sends a signal to the portable device to ring the alarm. This immediately informs the car owner about the theft thus overcoming the drawbacks of the existing tracking system. Once the thief starts the engine, the portable device tracks the car using GPS. Thus, the car owner can retrieve the car as soon as possible.

\section{BLOCK DIAGRAM}

The Block Diagram of the system is as shown in Figure 2. The system consists of the event tracking unit and a surveillance pad.

\subsection{Event Tracking Unit}

It consists of an asset tag and event detection module.

\subsubsection{Asset Tag}

It comprises of GPS receiver and the communication transreceiver.

\subsubsection{GPS Receiver}

The GPS receiver receives signals from satellites in global positioning satellite system. These signals are processed to identify the geographic location of the GPS receiver according to the well known GPS technology. The GPS receiver is powered by the battery. It is believed that a variety of known rechargeable batteries are preferred. However, reducing the size and weight of the battery also generally results in a reduction of the useful life of the battery. Therefore it is desirable that an asset tag operate in such a manner so as to conserve the consumption of the power by the GPS receiver.

\subsubsection{Communication Transreceiver}

It is a cellular-based transceiver used to receive Event Occurrence signal and transmit signals to the wireless network for ringing the alarm and enabling the display as per the event detected.

\subsubsection{Event Detection Module}

It consists of Door Sensor, Engine Sensor, Event detection logic and Signal Conditioning Electronics.

\subsubsection{Door Sensor}

It senses the locking and unlocking of the door. It sends the appropriate signal to the processor.

\subsubsection{Engine Sensor}

It senses the start and stop of the engine. It sends the appropriate signal to the processor.

\subsubsection{Event Detection Logic (Processor)}

It continuously checks the status of the sensors to set and clear Door Status bit (DS) and Engine Status bit (ES) accordingly. It also sends the Event Occurrence signal to the communication transreceiver.

\subsubsection{Signal Conditioning Electronics}

It converts the signal to the desired form with the help of transducers, amplifier and filters.

\subsection{Surveillance Pad}

The portable device mentioned above is termed as Surveillance Pad. It is carried by the owner to monitor the car. It consists of Wireless Network, Processing Unit, Alarm and Display.

\subsubsection{Wireless Network}

This network uses radio communication to communicate with the event tracking unit present in the car. The frequency ranges in Megahertz.

\subsubsection{Processing Unit}

It receives the event occurrence signal and rings the alarm when the door is opened. It also enables the display on receiving the position report when the engine starts.

\subsubsection{Alarm System and Display}

They are enabled by the processing unit. Display continuously shows the location of the car.

\section{WORKING}

AT Mega32 microcontroller is part of AVR series of microcontroller manufactured by Atmel with RISC architecture $32 \mathrm{~Kb}$ of in-system programmable Flash, 1K EPROM, 2K SRAM, 32 bit multifunction General purpose I/O, TWI, USART, SPI, JTAG interface support, etc. Atmel also provides free software support in the form of AVR ' $C$ ' compiler, AVR Studio for Software debugging and simulation in assembly language and Ponyprog software is also available for flash programming.

The interface diagram, of micro-controller system is shown in figure 3.

Port $\mathrm{C}$ is configured as input port and remaining ports are grounded. In this application, a mobile is connected to microcontroller based system through RS 232C data link cable. Internally RxD and TxD are connected to 9-pin RS232 female connector through MAX 232 IC for TTl-RS232C signal translation. PA0 is connected to potential divider circuit to provide variable analog voltage at its input. The crystal of $1.8432 \mathrm{MHz}$ is chosen for generating baud rate of $115.2 \mathrm{kbps}$ for serial communication with mobile.

AT Mega32 is initialized to configure port $\mathrm{C}$ as input. Universal Baud Rate Register (12 bit) UBRRG \& UBRRL are initialized to $000 \mathrm{H}$ for baud rate of $115.2 \mathrm{kbps}$. The frame format of 8 bit, no parity and 1 stop bit is set using Universal Control \& Status Register C (UCSRC). Receiver and transmitter are enabled through appropriate bits in UCSRB. For transmission, it is recommended to check the empty status of transmit buffer with the help of UDRE flag in UCSRA. If the transmit buffer is empty then data byte to be transmitted to TxD pin is sent through Universal Data Register (UDR). For serial data reception, $\mathrm{RxC}$ bit in UCSCRA is checked at regular intervals and if it is active, incoming data byte from $\mathrm{RxD}$ pins is read through UDR.

For enabling SMS reception from control system mobile, microcontroller sends

1. 'ATZ' command to reset modem of control system mobile.

2. 'AT' command to check modem status.

3. 'AT + CGMF' command to select to text mode.

4. 'AT + CNMI' command to set incoming SMS message indications. 


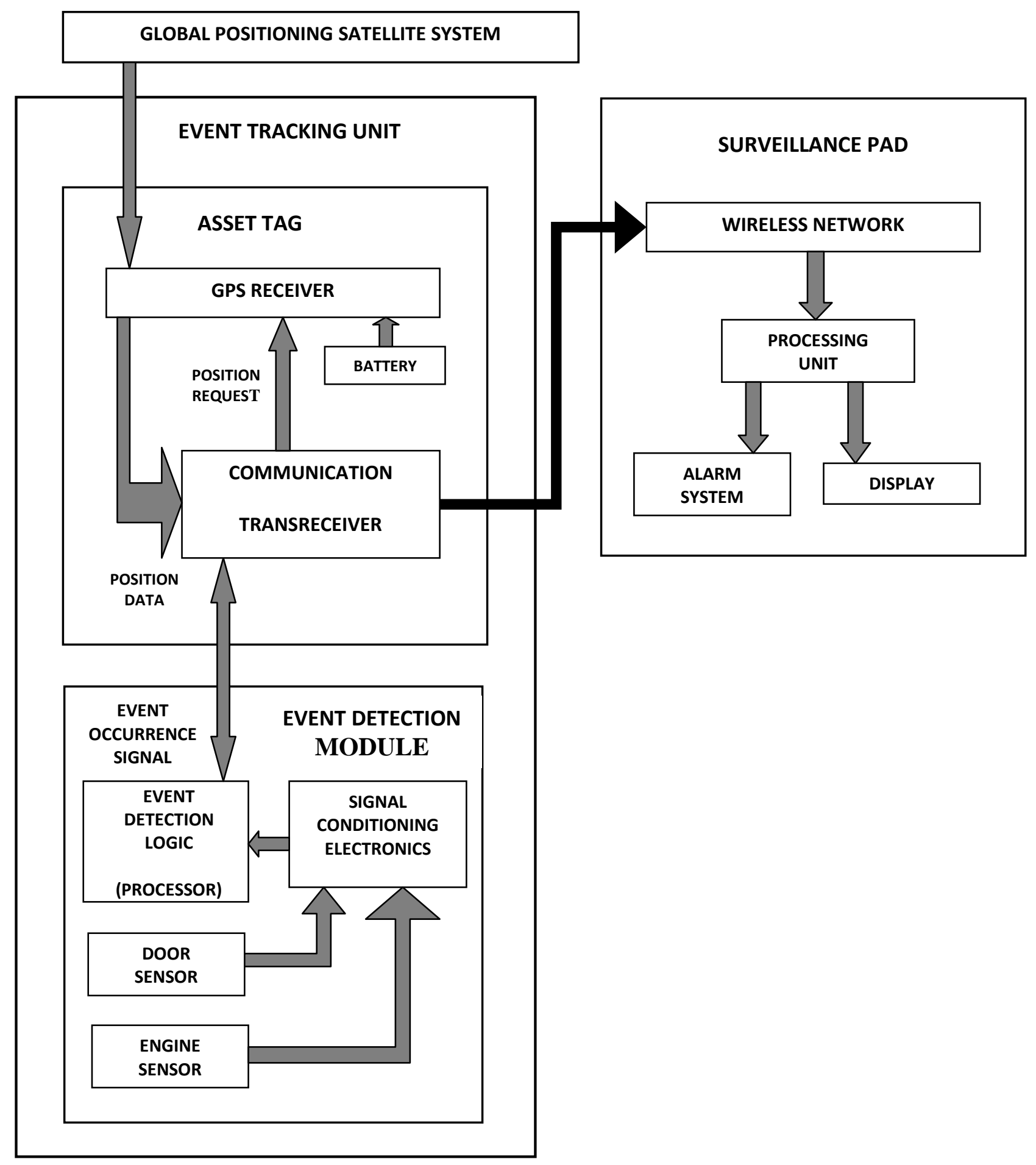

Figure 2: Block Diagram 


\section{MICROCONTROLLER}

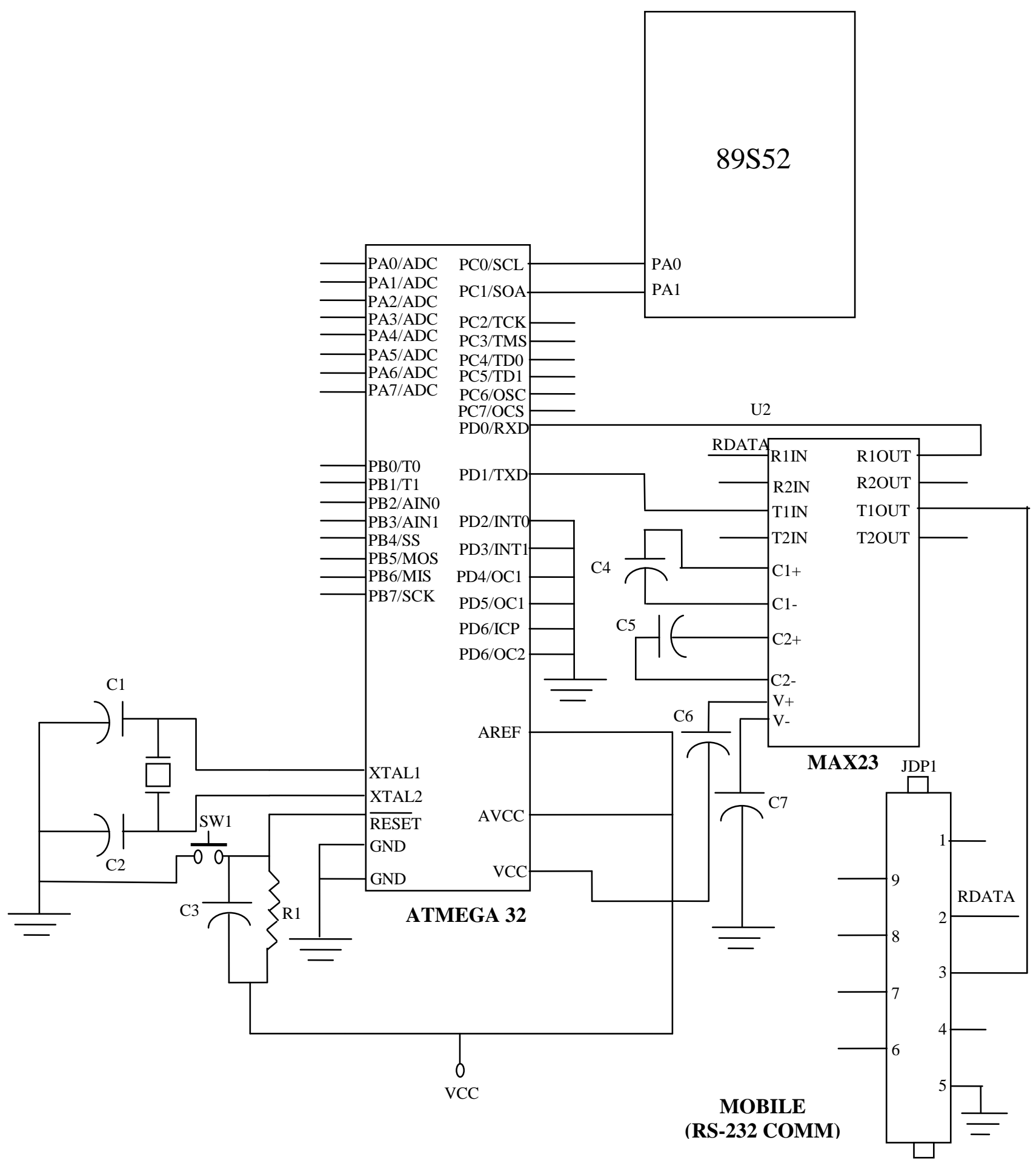

Figure 3: Interfacing Diagram

PA0 and PA1 of microcontroller are connected to PC0 and PC1 pins of AT Mega32. Microcontroller then checks digital input port 
bits and analog input at periodic intervals and sends SMS whenever there is change in status of input port bits or deviation of analog input from prescribed limits.

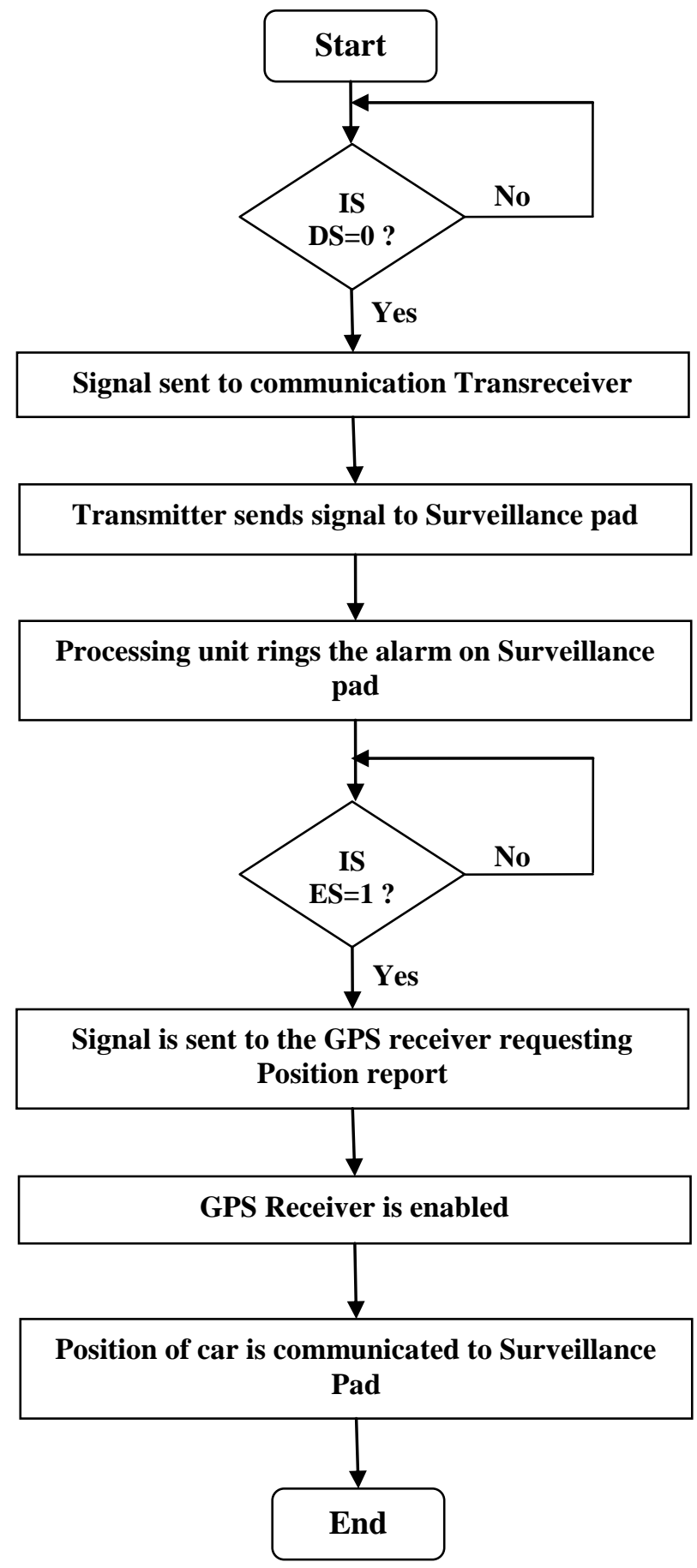

Figure 4: Flowchart of Detailed Process

The detailed description of the process shown in flowchart is as follows:

When the owner activates the system, the event detection module initially checks the door status bit, $\mathrm{DS}$. $\mathrm{DS}=0$ indicates that the door is unlocked and DS $=1$ indicates that the door is locked. The event detection module is programmed to constantly check the DS bit. As soon as DS clears, a signal is sent to the communication transreceiver. Transmitter sends a signal to the surveillance pad which immediately rings an alarm alerting the driver regarding the opening of car door. Once DS bit clears, the event detection module starts checking for the engine status bit ES. ES=0 indicates the engine is $\mathrm{OFF}$ and $\mathrm{ES}=1$ indicates the engine is $\mathrm{ON}$. As soon as ES bit sets, another signal is sent to GPS receiver requesting for the position report through communication transreceiver. This enables the GPS receiver. The asset tag provides a position report by the prompt provided by the event indicator sent to the communication transmitter by the event detection module. Upon such prompting, the position of the car is communicated to the wireless network through communication transreceiver and forwarded to the surveillance pad. The display is enabled by the processing unit and the driver easily tracks the car. The event prompt has an advantage that the GPS receiver remains dormant, thus conserving power, until prompted by the local communicator to provide a position report.

\section{CONCLUSION}

The event detection system proposed by us can be implemented in places like banks, defence, museums, research centres and various other high security places thus facilitating immediate detection and quick recovery of the stolen object.

\section{COMMENTS}

1. Even if the thief disables the GPS system after opening the car, an alert is inevitably sent to the car owner regarding the theft. Hence it serves as a foolproof system for car theft detection.

2. Since the GPS receiver is activated only after the engine is started, a lot of power is conserved.

3. The processor present in the car itself can be used since it requires very less memory eliminating the need for a dedicated processor.

4. We could have used an RFID tag instead of GPS. But GPS is relatively cheaper and provides continuous tracking.

5. We could have ANDed both the door unlocking and engine starting events. But this logic will not provide a security to the valuables present inside the car since the thief need not necessarily steal the car.

\section{RECOMMENDATIONS}

1. The surveillance pad can be replaced by an SMS alert and GPS tracking on mobile which eliminates the need to carry an additional device.

2. Also we can use cryptography to encrypt the transmitted signal and decrypt it at the surveillance pad, thus reducing the brute-force attacks.

3. Just as with car owners, homeowners also have a desire to recover lost or stolen household items having significant value. Thus a similar system can be implemented at homes where multiple events can be detected.

\section{REFERENCES}

[1] Koji Hasegawa, "GPS Receiver and GPS Reception Method", US Patent Application Publication, September 6, 2001. 
[2] Timothy Neher, Anthony LaRochelle, "Security Tracker", US Patent Application Publication, Jul 10, 2007.

[3] Zeineh, Rashid A., "Vehicular Safety System and Method", US Patent Application Publication, Dec 11, 2003.

[4] edaboard.com Forum for Electronics, available at http://www.edaboard.com/

[5] SatSleuth GPS Tracking Systems, available at, http://www.satsleuth.com/
[6] Tech

Ebooks,

available

at http://www.freetechebooks.com/

[7] Auto-Theft.Info, available at http://www.auto-theft.info/

[8] Jesse H. Tanner, "Vehicle location", US Patent Application Publication, September 12, 1995.

[9] SmartMotorist.com, available http://www.smartmotorist.com/ 